\title{
Effect of Structure on Strength of Agglomerates using Distinct Element Method
}

\author{
Tina Bonakdar* and Mojtaba Ghadiri \\ Institute of Particle Science \& Engineering, University of Leeds, Leeds, UK
}

\begin{abstract}
Knowledge of agglomerate strength is highly desirable for compression and tableting, dissolution and dispersion and mitigation of dust formation. The behaviour of agglomerates is affected by parameters such as density, agglomerate size, primary particle size, and interparticle bond strength. The method of agglomeration influences the evolution of structure, and this in turn affects its strength. Furthermore, the methods of strength characterisation, i.e. quasi-static or impact produce different results. To understand the role of structure and the influence of test method, agglomerate failure behaviour has been analysed by the use of the Distinct Element Method (DEM). We report on our work on the simulation of the breakage of the agglomerates for different porosities and impact conditions, where the role of impact speed and angle and type of contact bonding model have been evaluated. The adhesive contact model of JKR is used to form an agglomerate. The effect of the bonding level on the strength and size distribution of the clusters released as a result of failure has been investigated. This work also evaluates the effect of structure (porosity) on the strength of the agglomerates.
\end{abstract}

\section{Introduction}

Agglomerates may be formed in various ways and their mechanical strength depends on many factors due to the degree of freedom afforded by the primary particles. Weak agglomerates formed for example by spray-drying are prone to attrition, which has adverse effects on product quality, ability to process, dust formation and explosion hazards [1]. Therefore it is highly desirable to develop predictive ability for agglomerate breakage. One of the important factors, which has a strong influence on strength, is the agglomerate structure, yet the current modelling capability is not predictive. Quasi-static and impact behaviour differ markedly, and for the latter the velocity and impact angle dependence are not well understood [2]. For some structures, the impact strength depends mainly on the normal component of the impact speed, whilst for other structures the tangential component is also influential, but the factors affecting the difference are unknown [3]. In addition the functional dependence of the extent of breakage on impact speed is often a square power law, but this is not universal, again the source of difference not understood [4].

In this work we use the Distinct Element Method (DEM) to analyse the effect of agglomerate structure on the impact strength of agglomerates of elastic spheres that are bonded together by a contact adhesive force, following the JKR model [5]. A sensitivity analysis is carried out addressing the effects of the single particle properties (adhesion) and agglomerate structure (porosity) on the impact strength and size distribution of the clusters that are formed as a result of impact. This enables the underlying causes of variations in the dependency of impact speed and angle to be elucidated.

\section{Methodology}

Simulations are done using EDEM software, DEM Solutions, Edinburgh, UK. The primary particles are generated using six different particle types. The properties of all the particle types are the same, however they vary in particle size and number. The use of particle type makes it possible to vary porosity by removing some types from the structure. Particles are generated in a large space and a centripetal force is applied to bring them together to form an agglomerate. A relaxation step is applied after agglomerate formation in order to minimise the residual stresses within the agglomerate to avoid their possible effects on the breakage. The particles are then bonded together using the JKR contact adhesion model. Six different surface energies are used in order to make the agglomerates $(0.5,1,1.5,2,3$ and 5 $\mathrm{J} / \mathrm{m}^{2}$ ). The agglomerates are then impacted at different impact velocities $(0.2,0.5,1,5,10$ and $20 \mathrm{~m} / \mathrm{s})$. Two impact angles are used (normal and inclined). The same impact tests are repeated for agglomerates with three different levels of porosity. The impact reuslts are then analysed using the concept of damage ratio $[6,7]$, which is defined as the ratio of number of broken contacts to the initial number of contacts, to explore the effects of above-mentioned parameters on the impact strength of agglomerates.

\footnotetext{
Corresponding author: t.bonakdar@,leeds.ac.uk
} 


\section{Results and Discussion}

Six agglomerates with different surface energies have been impacted at a range of impact velocities. The damage ratio as a function of impact speed is shown in Fig. 1.

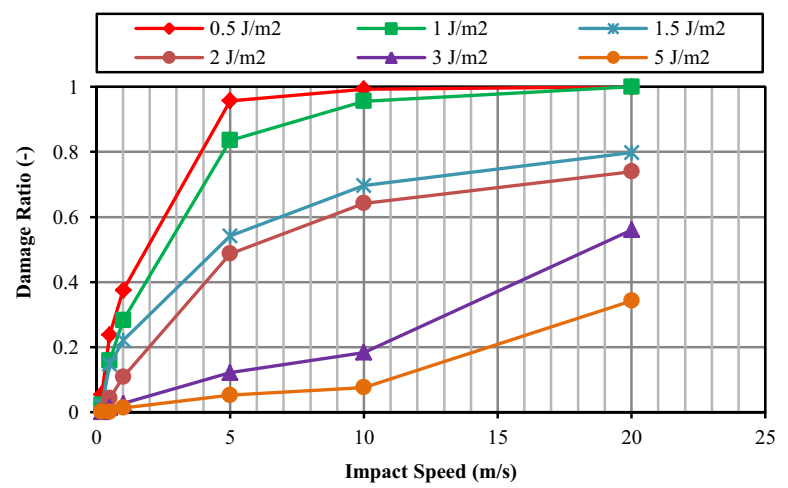

Fig. 1. Damage ratio as a function of impact speed for agglomerates with different cohesive interparticle forces

For a given surface energy, as the impact speed is increased, the damage ratio increases. At a given impact speed, the agglomerate with a lower surface energy breaks to a larger extent compared to the one with higher surface energy, which is as expected. However, three different trends are clearly observed, which are attributed to the pattern of breakage.

It is expected that the damage ratio follows a square of velocity relationship. Therefore the damage ratio has been plotted as a function of $V^{2}$ to see if such a dependency holds. Based on the results, a linear relationship is only observed for impact results of high surface energy agglomerates, but there is no such a dependency for low surface energy case (not shown here).

To explore the effect of impact angle on the extent of breakage, the agglomerates have been impacted at $60^{\circ}$ and $90^{\circ}$ (the impact angle is defined as the angle between agglomerate moving direction and the surface of the impact target). As an example the damage ratio as a function of impact speed is shown in Fig. 2 for impacting the agglomerate with $3 \mathrm{~J} / \mathrm{m}^{2}$ surface energy at these two angles.

Normal impacts cause more damage as compared to inclined ones. However, if the normal velocity component of the inclined impact is taken into account, then the data overlap, indicating that the agglomerate breakage is mainly dependent on the normal component (as shown in Fig. 3).

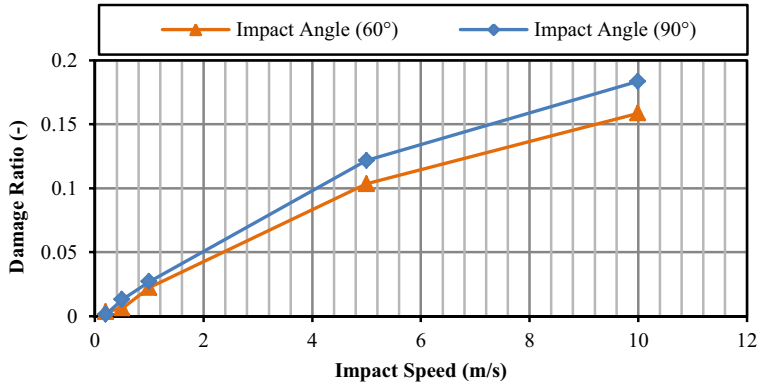

Fig. 2. Damage ratio as a function of impact speed for the same agglomerates (surface energy of $3 \mathrm{~J} / \mathrm{m}^{2}$ ) at different impact angles

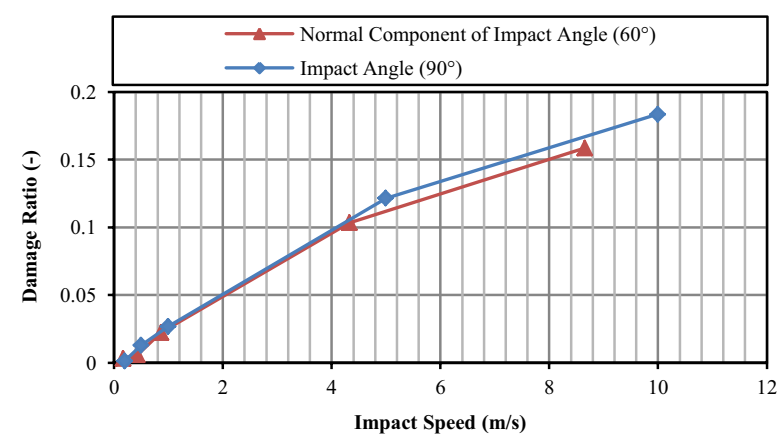

Fig. 3. Damage ratio as a function of normal component of the impact speed for the same agglomerates (surface energy of 3 $\mathrm{J} / \mathrm{m}^{2}$ ) at different impact angles

The effect of porosity on impact breakage has been studied by removing some particle types to make cavities inside the agglomerate. The impact breakage results of three cases, reference agglomerate (referring to the initial agglomerate) and more porous agglomerates (51\% and $61 \%$ porosity) are shown in Fig. 4.

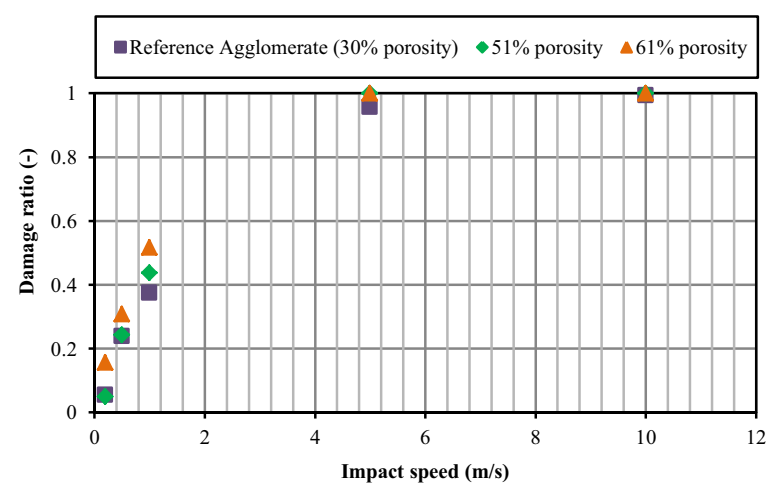

Fig. 4. Damage ratio as a function of impact speed for three agglomerates with different levels of porosity and surface energy of $0.5 \mathrm{~J} / \mathrm{m}^{2}$

For the three porosity levels analysed here, there are no notable differences in the damage ratio for all the impact velocities. Interestingly, applying the same analysis to the agglomerates with high surface energy shows a huge 
difference in damage ratio for three different porosities (as shown in Fig. 5). More work is needed to map out the breakage patterns in terms of these two factors.

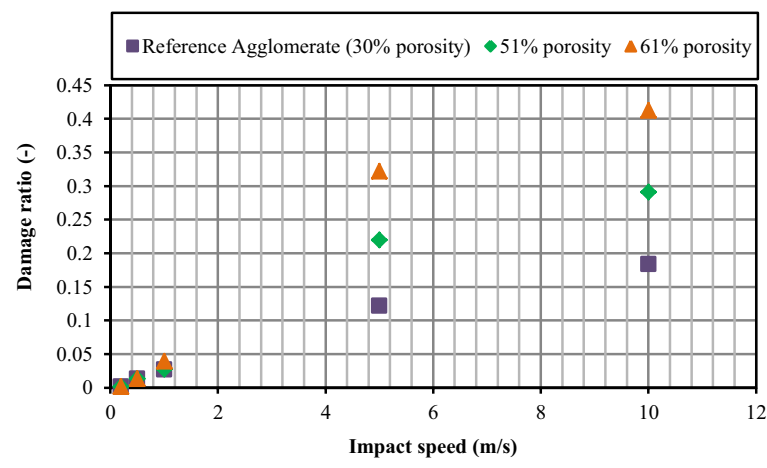

Fig. 5. Damage ratio as a function of impact speed for three agglomerates with different levels of porosity and surface energy of $3 \mathrm{~J} / \mathrm{m}^{2}$

The damage ratio for the above conditions was analysed as a function of impact speed. However, the breakage of contacts can also be related to Weber Number (We). Previously Kafui and Thornton [6] used this approach to analyse the agglomerate breakage. Weber number is defined based on the equation below,

$W e=\frac{\rho D V^{2}}{\Gamma}$

where $D$ and $\rho$ are particle diameter and density of the particles, respectively. $V$ is the impact speed. However, the modified version of the Weber number ( $\left.\mathrm{We}^{\prime}\right)$ as proposed by Thornton et al. [7] is more accurate, as it considers the speed below which there is no breakage $\left(V_{0}\right)$.

$W e^{\prime}=\frac{\rho D\left(V-V_{0}\right)^{2}}{\Gamma}$

Moreno-Atanasio and Ghadiri [8] did an extensive piece of work on impact breakage of spherical agglomerate using DEM simulation. Based on their model, the number of broken contacts is proportional to the incident kinetic energy. By using an energy balance between the work required to break interparticle contacts and incident kinetic energy, they proposed the following relationship between damage ratio $(\Delta)$ and the surface energy,

$\Delta \propto W e^{\prime}\left(\frac{E D}{\Gamma}\right)^{2 / 3}$

where $E$ is the primary particle elastic modulus. Therefore, the simulation data have been analysed using Eq. (3) to explore the dependency of damage ratio on the surface energy as shown in Fig. 6. Based on their work, a unification is expected when the damage ratio is plotted as a Weber-dependant group. However, this is not the case. The trend observed in Fig. 6 is similar to that of Fig. 1. In both Figures, there are three clear trends. Each trend is attributed to a different pattern of breakage. Based on the observation of the agglomerates after impact, impacting the agglomerates with low surface energies $\left(0.5\right.$ and $\left.1 \mathrm{~J} / \mathrm{m}^{2}\right)$ would result in disintegration, agglomerates with surface energies of 1.5 and $2 \mathrm{~J} / \mathrm{m}^{2}$ would result in fragmentation whilst for agglomerates with high surface energies ( 3 and $5 \mathrm{~J} / \mathrm{m}^{2}$ ) the damage is localised and chipping is the main pattern of breakage for these range of velocities. As an example the force distribution after impact at $0.5 \mathrm{~m} / \mathrm{s}$ is shown in Figs 7 and 8 for agglomerates with 0.5 and $3 \mathrm{~J} / \mathrm{m}^{2}$ surface energy, respectively. Here the lines connecting the spheres are shown with their colours representing the magnitude of the contact force.

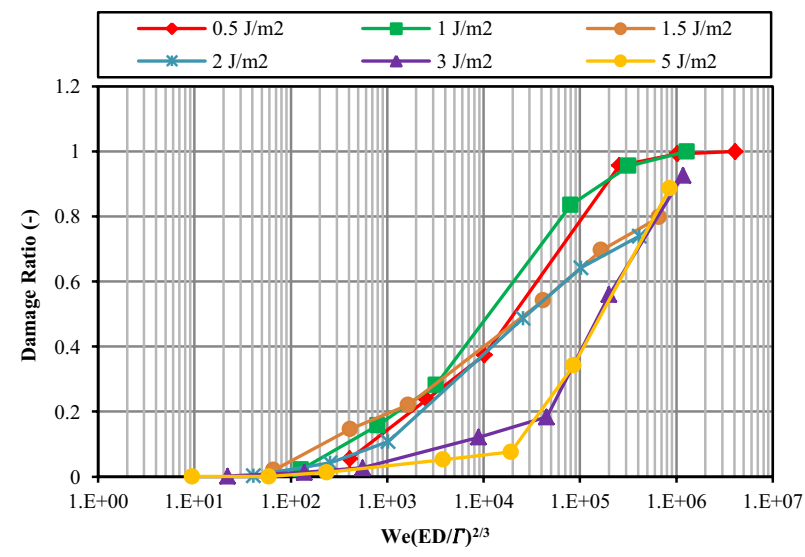

Fig. 6. Damage ratio as a function of $\Gamma \mathrm{E} / \mathrm{q}$.ff7with different surface energies

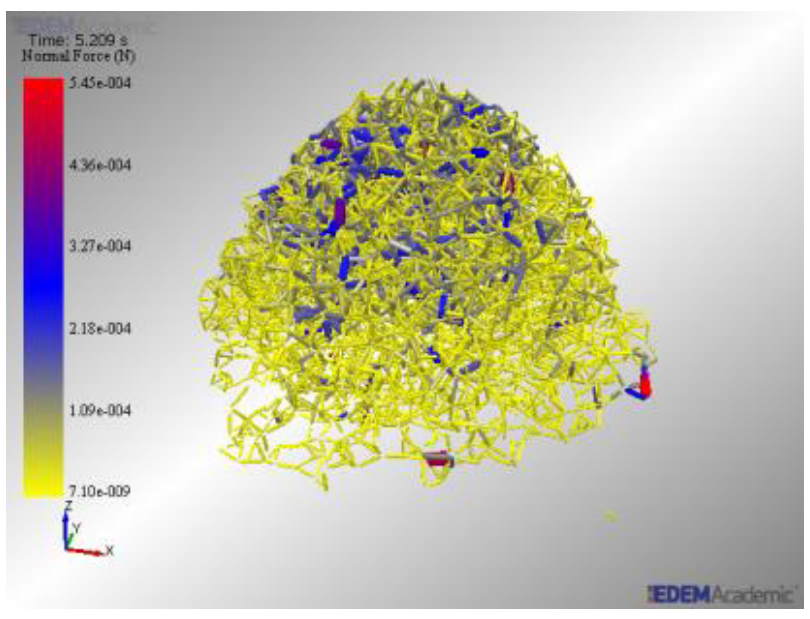

Fig. 7. Side view of force distribution after impacting at 0.5 $\mathrm{m} / \mathrm{s}$ for agglomerates with $0.5 \mathrm{~J} / \mathrm{m}^{2}$ surface energy 


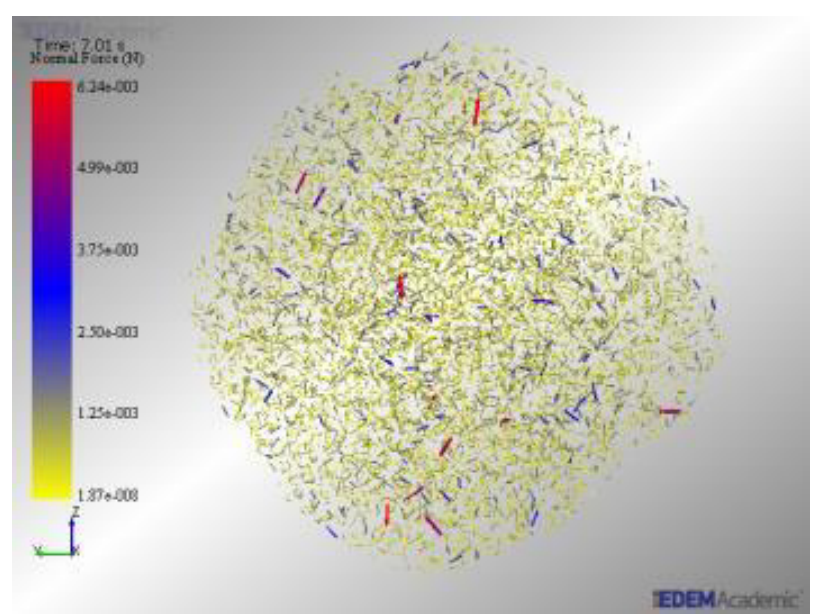

Fig. 8. Side view of force distribution after impacting at 0.5 $\mathrm{m} / \mathrm{s}$ for agglomerates with $3 \mathrm{~J} / \mathrm{m}^{2}$ surface energy

The force distributions are exported after impact at 0.5 $\mathrm{m} / \mathrm{s}$. Side views of agglomerates with the surface energy of $0.5 \mathrm{~J} / \mathrm{m}^{2}$ and $3 \mathrm{~J} / \mathrm{m}^{2}$ are shown in Figs 7 and 8 , respectively, where the impact side is at the base of the agglomerate. However, the data for both agglomerates have been exported at $0.01 \mathrm{~s}$ after impact, and the difference in the time scale shown in Fig. 7 is due to the time taken for agglomerate generation and applying surface energy at previous steps before impact. The disintegration of the agglomerate with $0.5 \mathrm{~J} / \mathrm{m}^{2}$ surface energy is clearly seen, as the bottom part of the agglomerate (close to the impact zone) is completely deformed and contacts broken in the impact zone and beyond. The force has also propagated within the agglomerate and reached to the top part, as shown in Fig. 7 , causing breakage of the contacts. A different scenario is observed in Fig. 8 for $3 \mathrm{~J} / \mathrm{m}^{2}$ surface energy. There is no clear deformation at the impact zone (bottom part of the agglomerate) with little/ no contact breakage, and the force distribution has a different pattern as compared to Fig. 7.

Based on these observations, the lack of unification of breakage data in Fig. 6 is presumably due to different patterns of breakage. For tested agglomerates by Moreno-Atanasio, all of them failed through the same pattern of breakage, for which a good unification was obtained, as the damage was ratio plotted as a function of $W e^{\prime}\left(\frac{E D}{\Gamma}\right)^{2 / 3}$. However, more probing of the differences in the method of agglomeration preparation is required to explore the underlying differences in the breakage behaviour of the two systems.

\section{Conclusions}

Agglomerate structure affects its strength. Impacting the agglomerates with different surface energies for a range of impact velocities shows three different trends of breakage, which are attributed to different patterns of breakage. The agglomerates with higher surface energy undergo less breakage compared to the agglomerates with lower surface energy, as intuitively expected. Impacting the agglomerates at the normal angle would result in a larger number of broken contacts compared to impact at inclined angles. It has been found, for the range of conditions analysed here, that the agglomerate failure is mainly dependent on the normal component of the impact speed. Three different levels of porosity have been used to explore the effect of porosity on the impact strength of agglomerates. Generally, more porous agglomerates break to a higher extent compared to less porous agglomerates, the difference being more pronounced for agglomerates with high surface energy. As an ongoing work, the newly developed bonding model of Brown et al. [9] is used to simulate the impact breakage of agglomerates. The model is based on the Timoshenko Beam Bond Model (TBBM). This provides a more realistic representation of granules made using a liquid binder which on drying forms a solid bond.

\section{References}

1. T. Bonakdar, M. Ghadiri, H. Ahmadian, L. Martin de Juan, H. Tantawy, H. D, Smith, Impact attrition of spray-dried burkeite particles. Powder Technol. 304. 1-7 (2016)

2. A. Samimi, R. Moreno, M. Ghadiri, M. Analysis of impact damage of agglomerates: Effect of impact angle. Powder Technol. 143-144, 97-109 (2004)

3. A. D. Salman, M. J. Hounslow, J. P. K. Seville. Granulation handbook. 11 (2007)

4. J. Subero, M. Ghadiri, Breakage patterns of agglomerates. Powder Technol. 120, 3 (2001)

5. K. L. Johnson, K. Kendall, A.D. Roberts, Surface energy and the contact of elastic solids. Proceedings of the Royal Society of London A., 324, 301-313 (1971)

6. K. Kafui, C. Thornton, Computer simulated impact of agglomerates. Powders and Grains (1993)

7. C. Thornton, K. Yin, M. Adams, 1996. Numerical simulation of the impact fracture and fragmentation of agglomerates. J. Phys. D Appl. Phys. 29. 424435 (1996)

8. R. Moreno-Atanasio, M. Ghadiri, M. 2006. Mechanistic analysis and computer simulation of impact breakage of agglomerates: Effect of surface energy. Chem. Eng. Sci. 61, 8 (2006)

9. N. J. Brown, J. F. Chen, J. Y. and Ooi, J.Y. 2014. A bond model for DEM simulation of cementitious materials and deformable structures. Granular Matter. 16, 3 (2014) 